\title{
POINCARE PLOT OF HEART RATE VARIABILITY: QUANTITATIVE ANALYSIS OF SYMPATHETIC NERVOUS ACTIVITY IN NON-OBESE POLYCYSTIC OVARY SYNDROME PATIENTS
}

\author{
Malathi Balamurugan'1, Balamurugan², Gomathi Ramanathan ${ }^{3}$ \\ ${ }^{1}$ Assistant Professor, Department of Physiology, Karuna Medical College, Chittur. \\ ${ }^{2}$ Chief Consultant and Medical Director, Vadavalli Medical Centre, Coimbatore. \\ ${ }_{3}^{3}$ GR Hospitals Post Graduate Medical and Research Institute, Coimbatore.
}

\begin{tabular}{l}
\hline ABSTRACT \\
BACKGROUND \\
Polycystic Ovary Syndrome (PCOS) is one of the most common endocrinopathy in premenopausal women. \\
AIM \\
The aim of the study was to evaluate the effectiveness of the Poincare plot analysis of Heart Rate Variability (HRV) in PCOS. \\
METHODS AND MATERIALS \\
24 PCOS diagnosed by Rotterdam 2003 Diagnostic Criteria and were of lean and ideal weight as per WHO criteria and 24 BMI \\
matched, age matched normally menstruating women served as study participants. People of the study group underwent 5 min of \\
ECG, which was evaluated for HRV. HRV analysed were Geometrical parameters (HRV, TRI, INDEX, TINN), Total Power (TP) and \\
Poincare plot parameters (SD1, SD2, SD1/SD2, S).
\end{tabular}

\section{RESULTS}

The Poincare scatter grams were narrower in patients and wider in control groups showing parasympathetic withdrawal and sympathetic dominance, but were not statistically significant. Area (S), TP and HRV TRI INDEX, TINN showed overall decrease in autonomic activity denoting altered sympathovagal balance favouring sympathetic dominance. There was a significant correlation of TP, SD1, SD2, S, TINN and HRV TRI INDEX with increased Rate Pressure Product (RPP) as well as with one another, but not with BMI. The regression analysis did not lay forward the independent associations of these variables.

\section{DISCUSSION AND CONCLUSION}

This study indicates the total variability is decreased even in young, lean and ideal weight PCOS patients. Larger studies are needed to evaluating the short- and long-term variability.

\section{KEYWORDS}

Polycystic Ovarian Syndrome, Heart Rate Variability, Poincare Plot Analysis, Geometrical Parameters, Cardiac Autonomic Innervations, Sympathovagal Activity.

HOW TO CITE THIS ARTICLE: Balamurugan M, Balamurugan, Ramanathan G. Poincare plot of heart rate variability: quantitative analysis of sympathetic nervous activity in non-obese polycystic ovary syndrome patients. J. Evolution Med. Dent. Sci. 2016;5(47): 3005-3010, DOI: 10.14260/jemds/2016/700

\section{INTRODUCTION}

Polycystic Ovary Syndrome (PCOS) is one of the most common endocrinopathy of premenopausal women. It was first described by Stein Leventhal in 1935 with a prevalence rate of $25 \%$.(1) of the Indian population. PCOS is a clinical combination of anovulation and hyperandrogenism; now getting attention as a new face of metabolic syndrome.(2)

The cardiovascular involvement in PCOS has been investigated and many reported some abnormalities. The data regarding the cardiovascular mortality and morbidity are conflicting.(3) PCOS patient have an estimated 4-11 fold increased risk for coronary artery disease, based on the prevalence of risk factors.(4) There was high prevalence of diabetes (40\%) and hypertension (60\%).(5)

The abnormalities found were decreased cardiac systolic flow velocity.(6)

Financial or Other, Competing Interest: None.

Submission 11-04-2016, Peer Review 20-05-2016,

Acceptance 26-05-2016, Published 13-06-2016.

Corresponding Author:

Dr. Malathi Balamurugan,

Assistant Professor,

Karuna Medical College,

Vilayodi, Chittur.

E-mail:dr_balamala@yahoo.com,dr_malu@rediffmail.com

DOI: $10.14260 /$ jemds $/ 2016 / 700$ diastolic dysfunction.(7) increased vascular stiffness.(8) endothelial dysfunction.(9) low-grade chronic inflammation.(10) increased homocysteine.(11) impaired fibrinolysis.(12) and increased tissue plasminogen activator antigen.(13)

Obesity has been associated with Cardiovascular (CV) autonomic dysfunction in the form of increased sympathetic activity.(14) It was seen that obesity factor was just a factor modifying this syndrome.(15) and metabolic syndrome was prevalent in even lean PCOS.(15)

A heart rate variability measurement from electrocardiographic recording has been shown to be useful to assess cardiac autonomic function.(16) However, in our previous study we found the linear measures of HRV were affected even in non-obese young PCOS with unfavourable lipid profile.(17)

Analysis of the non-linear dynamics of HRV would enable a better physiological interpretation of the HRV and for the assessment of the risk of sudden death.(16) The analysis of Poincare plots or sections of RR intervals is an emerging method of non-linear dynamics applied in HRV analysis.(18)

Poincare plot of RR intervals is an useful visual tool which is capable of summarizing an entire RR time series derived from an Electrocardiogram (ECG) in one picture and a quantitative technique, which gives information on the long- 
and short-term HRV.(19) However, no studies have examined the non-linear component of HRV in PCOS excluding the obesity factor.

Therefore, our aim was to evaluate the effectiveness of the Poincare plot analysis of Heart Rate Variability (HRV) in nonobese young PCOS patient and to compare them with regularly menstruating apparently healthy non-obese controls. We hypothesized that the "PCOS is associated with alterations of short- and long-term variability of Poincare plot parameters."

\section{METHODOLOGY}

This was a case control study conducted in the Department of Physiology, PSG IMS and R. Both study and control groups gave written informed consent. Clearance from the Institute's Human Ethics Committee was got. The patient study group included PCOS, who were diagnosed with physical findings of hyperandrogenism, oligo/anovulation and ultrasonography, after exclusion of specific ovarian, adrenal and pituitary disorders according to Rotterdam 2003 diagnostic criteria.(20)

\section{Sample Size Calculation}

According to the disease prevalence in India, the sample size was calculated.(1) The required sample size calculated was 18 . We analysed 25 patients, of which one was excluded because of presence of ectopic beats.

\section{SUBJECTS}

\section{a. Patient Group}

24 non-pregnant ideal and lean weight (Measured by BMI Body Mass Index) women with PCOS. The patients were grouped as lean and normal as per the WHO criteria.(21)

\section{b. Control Group}

24 regularly menstruating (Every 27-32 days) volunteer medical students, doctors, nurses and staff of the hospital and who were matched for age and BMI were included.

Inclusion criteria for both groups included young women aged 16 to 35; they were lean or ideal weight according to BMI and $\mathrm{W} / \mathrm{H}$ ratio, waist circumference less than $<80 \mathrm{cms}$; they were not on any medications affecting lipid or carbohydrate metabolism at least for past 2 months. Exclusion criteria included women below 16 and above 35, pregnant and lactating women, menopausal women, those who had undergone hysterectomy, women taking lipid lowering drugs, oral hypoglycaemic drugs or insulin sensitizing agents, oral contraceptives and sex steroids for last 2 months and those patients were on current infertility treatment were excluded. Control group were in their follicular phase (6-8 days the start of menstruation) and PCOS were amenorrhoeic during data recording. The study group had their relevant laboratory investigation. The anthropometric parameters included were.

\section{a. Body Mass Index (BMI)}

The study group were measured for height and weight (wt). Height was measured in cms. The study participants stood in their upright position using the Height measuring scale. The weight was measured using electronic weighing machine. From this BMI was obtained by dividing weight in Kg by square of the height (In meters). They were selected according to World Health Organization. Obesity is graded as underweight (BMI $\left.<18.5 \mathrm{~kg} / \mathrm{m}^{2}\right)$, normal $(18.5-24.9 \mathrm{~kg} / \mathrm{m} 2)$, pre-obese $(25.0-29.9 \mathrm{~kg} / \mathrm{m} 2)$, and class I obese (30.0-34.9 kg/m2) (21).

\section{b. Waist Circumference}

Waist circumference was taken by standard measures.(22) The subject assuming a standing position and then the points were marked on the subjects. Waist circumference was measured half way between the lower border of the ribs and the iliac crest in the horizontal plane. 2 measurements to the nearest $0.5 \mathrm{~cm}$ was measured. A third measurement was taken if the variation between the measurements were $>2 \mathrm{cms}$. The mean of the 2 closest measurements was calculated. For females, waist circumference $80-87.9 \mathrm{cms}$ was graded as overweight and $\geq 102 \mathrm{cms}$ as obese.

\section{c. Waist Hip Ratio (WHR)}

We also obtained standard cut-off for WHR, which denotes risk ( $>0.85$ in women) and lower cut-offs (0.80 in women).(23)

\section{CARDIOVASCULAR PARAMETERS}

\section{a. Basal Heart Rate (BHR)}

After 5 min of sitting at rest by palpating the left radial artery at wrist, the pulse rate was counted for complete 1 minute.

\section{b. Resting Blood Pressure (RBP)}

After 20 minutes of quiet supine rest, blood pressure was recorded. Subjects were in supine position using a manual sphygmomanometer (A Novaphone make). Systolic and diastolic blood pressure was measured. Recorded from right arm to the nearest $2 \mathrm{mmHg}$. Blood pressure was defined as the points of appearance and disappearance of Korotkoff sounds respectively.

\section{c. Rate-Pressure Product (RPP)}

RPP, a determinant of myocardial oxygen consumption and workload was calculated using the formula.(24)

$\mathrm{RPP}=(\mathrm{BHR} \times \mathrm{SBP}) \times 10^{-2}$

\section{HRV Analysis}

Task force guidelines on HRV analysis were followed. Morning 2-4 hours after taking breakfast, the tests were done. The 5 min ECG recording was taken from the study group. They were in lying position, quiet in a dark room for 20 minutes with open eyes, not falling asleep and not making any movements. This ECG was used for the calculation of mean heart rate and HRV during rest in the supine position.

Lead II ECG was recorded by placing three disposable adhesion electrodes on the limb. Baseline electrocardiograms were obtained from all subjects. Participants with abnormal baseline ECG (Including juvenile pattern) and ectopic beats were excluded.

ECG was obtained by the students Biopac version 1.3. RR intervals were obtained after clearance of noise and baseline fluctuations by digital filters. The data was filtered using a digital notch filters with a sampling rate of 1000 samples/sec. The inbuilt software selected the RR peaks and these RR intervals, which were obtained as time points were then fed into a Microsoft Excel Sheet and RR intervals were copied to a notepad file.

HRV analysis software, version 1.1 from Biomedical Signal Analysis group, Department of Applied Physics, University of Kuopio, Finland was used to do the HRV analysis. To analyse data lengths of 5 minutes, 256 seconds of RR interval data was taken from the tachogram and interpolated at $4 \mathrm{~Hz}$ to get 1024 points. Power spectral analysis was done by Fast Fourier 
Transformation (FFT) after de-trending and removal of the mean from the data points. Hanning window was applied to prevent spectral leakage (Default). The power spectral density was obtained by Welch's periodogram, using window width of 512 data points with an overlap of 256 points. Both linear and non-linear dynamics were obtained. Linear dynamics were analysed in our previous study.(17) Here, we focus on geometric methods of linear dynamics as well as non-linear HRV parameters.

\section{Linear Dynamics (Geometric Methods)}

This is one of the standard methods for analysing the HRV (1996, task force). This includes,

HRV triangular index, which is the integral of the density distribution (i.e. the number of all NN intervals) divided by the maximum of the density distribution.

Triangular interpolation of $\mathrm{NN}$ Interpolation of $\mathrm{NN}$ Interval Histogram (TINN), which is the baseline width of the minimum square difference triangular interpolation of the highest peak of the histogram of all NN intervals.(16)

\section{Linear Dynamics}

(Frequency Domain)

Total power (TP = LF + HF power): In short term recordings (Say 5 minutes), this represents the total heart rate variability which includes the variability of both the sympathetic and the parasympathetic components.

\section{Non-linear Dynamics: Using Poincare Plot}

Poincare plot is a visual presentation of time series signal to recognize the hidden patterns. It is a two-dimensional graphic representation of the correlation between consecutive RR intervals, in which each interval is plotted against the following interval. It is a qualitative tool. By assessing the shape formed, degree of complexity of RR intervals can be analysed. It is an ellipse and the dispersion along the major and minor axis of the ellipse is measured.(25)

The standard deviations of the instantaneous and longterm R-R interval variability are calculated. In the scatter diagram of the new axis, the dispersion of the points around the $\mathrm{x} 2$ axis is measured by the standard deviation denoted by SD1. This quantity measures the width of the Poincare cloud and therefore indicates the level of short-term HRV. The length of the cloud along the line-of-identity measures the long-term HRV and is measured by SD2, which is the standard deviation around the long axis.(26) we acquired.

\section{There are Two Standard Descriptors of Poincare Plot Namely}

a. Standard deviation 1 (SD1): It is the Standard Deviation (SD) of the instantaneous (Short-term) beat-to-beat R-R interval variability (minor axis of the ellipse or SD1).

b. Standard deviation 2 (SD2): It is the SD of the long-term $\mathrm{R}-\mathrm{R}$ interval variability (major axis of the ellipse or SD2).

c. SD1/SD2: Ratio of the standard deviations.

d. Area of the ellipse (S): It is the amount of area covered by the ellipse. Calculated by doing the product of $\pi$, SD1 and SD2. It represents total HRV.(27)

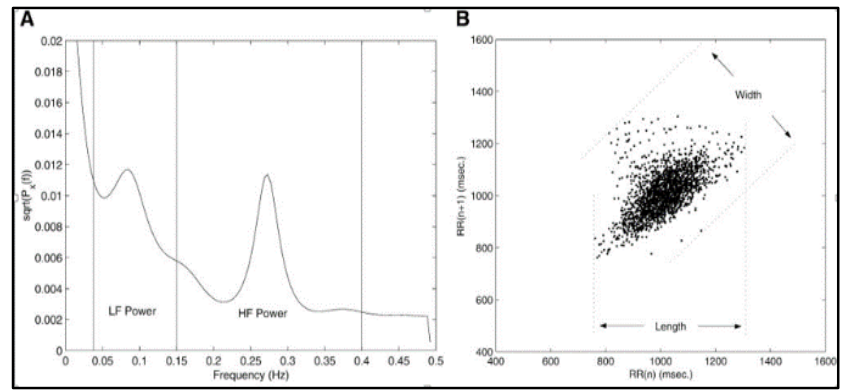

Fig. 1: The Poincare Plot SD1 and SD2

\section{Statistical Analysis}

The Statistical Package for the Social Science (SPSS 19.0 version for windows) was used for statistical analysis. Independent sample ' $t$ ' test was used to compare the measured parameters of patients with PCOS and control group. Pearson's correlation analysis was used to investigate the relationship between BMI, RPP and HRV parameters. Multiple regression analysis was also done to assess the contribution of individual factors to RPP and BMI. P $<0.05$ was considered statistically significant.

\section{RESULTS}

Values were expressed as Mean \pm SD.

\section{Basic Clinical Data}

The study group are the same subject we included for our previous work.(17) hence their basic parameters were the same. PCOS and control were of same age group $(22.96 \pm 3.96$ vs $24.21 \pm 4.69)$, BMI $(22.12 \pm 2.56$ vs $20.86 \pm 2.73)$ and weight (53.60 \pm 8.86 vs $51.09 \pm 9.31$ ) with $P$ value $0.324,0.104$ and 0.344 respectively. Resting blood pressure showed SBP $107.67 \pm 10.66$ vs $106.17 \pm 14.30$ and DBP $(73.58 \pm 8.75$ vs $71.08 \pm 9.14$ ) with $P$ value 0.682 and 0.338 respectively was not significant. RPP $(89.035 \pm 15.478$ vs $78.85 \pm 14.52)$ and BHR $(82.65 \pm 10.87$ vs $74.00 \pm 6.78)$ was statistically significant with p value 0.02 and 0.01 respectively (Refer Table 1; Fig. 2).

Poincare plot analysis showed SD1 SD2 SD1/SD2 were not significant with $\mathrm{p}$ value $0.322,0.546$ and 0.559 respectively. (Refer Table 1; Fig. 2) Total area $\mathrm{S}$ was statistically significant $(\mathrm{p}=0.026)$ (Refer Table 1; Fig. 3). Of the geometrical parameters HRV TRI INDEX, TINN both were statistically significant with $p$ value 0.008 and 0.016 respectively. Linear parameter TP was also statistically significant $(\mathrm{p}=0.018)$ (Refer Table 2; Fig. 4).

All the HRV parameters (SD2, SD1/SD2, S, TRI INDEX, TINN and TP) analysed had a statistically significant negative correlation with RPP with $\mathrm{p}$ value $<0.001,<0.001,<0.001$, $<0.001,<0.001$ and 0.004 respectively, except SD1 which had a significant positive correlation ( $P$ value $<0.001$ ). There was no significant correlation between HRV parameters and BMI. Also BMI and RPP was not correlated. (Refer Table 3). The geometrical parameters correlated significantly with TP and Poincare data S with P value $<0.001$ (Refer Table 4). Regression analysis showed no significant association between HRV parameters with RPP and BMI. (Refer Table 5). 


\begin{tabular}{|c|c|c|c|}
\hline Parameter & Case & Control & P value \\
\hline SD1 & $24.93 \pm 14.37$ & $39.19 \pm 16.46$ & 0.322 \\
\hline SD2 & $65.23 \pm 19.51$ & $55.14 \pm 26.84$ & 0.546 \\
\hline SD1/SD2 & $0.45 \pm 0.19$ & $0.59 \pm 0.25$ & 0.559 \\
\hline S & $5290.34 \pm 5067$. & $8723.58 \pm 525$ & 1.46 \\
RPP & 83 & 0.026 \\
\hline Table 1: Comparison of RPP and Poincare Plot Analysis \\
of the PCOS and Control Group \\
\hline
\end{tabular}

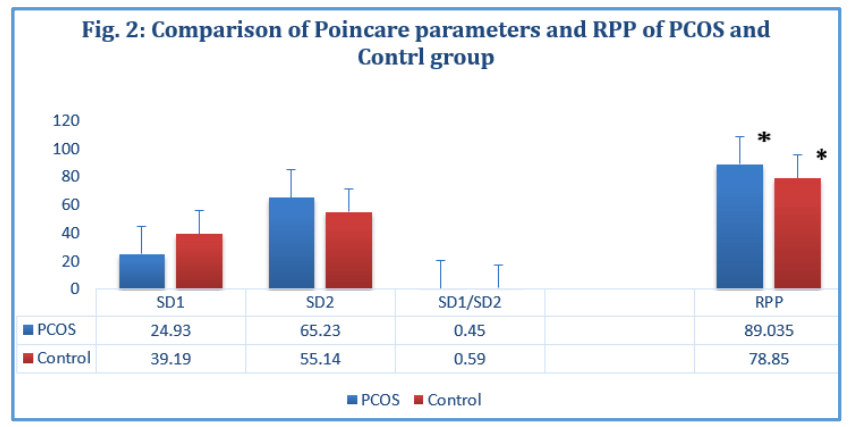

Fig. 2: SD1- Standard Deviation 1 (short-term variability), SD2-Standard Deviation 2 (long-term variability), SD1/SD2-Ratio, RPP - Rate Pressure Product. *-Significant

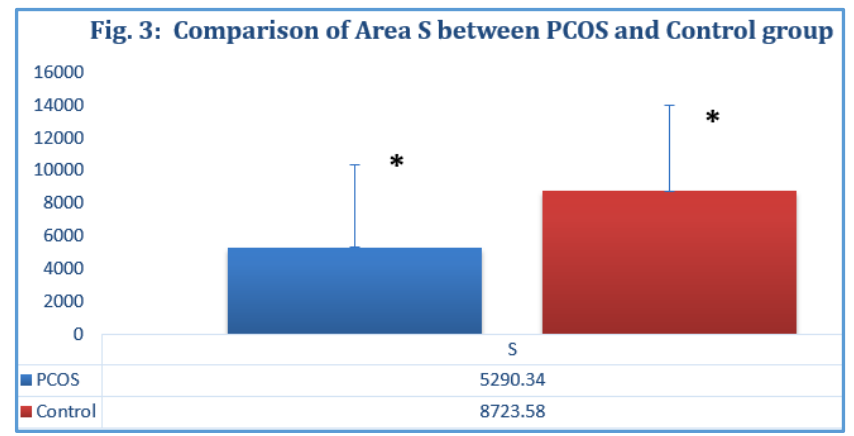

Fig. 3: S- Area S (Total HRV Variability) *-Significant

\begin{tabular}{|c|c|c|c|}
\hline Parameter & Case & Control & $\begin{array}{c}\text { P } \\
\text { value }\end{array}$ \\
\hline $\begin{array}{c}\text { HRV TRI } \\
\text { INDEX }\end{array}$ & $0.0671 \pm 0.0275$ & $0.0901 \pm 0.0295$ & 0.008 \\
\hline TINN & $183.125 \pm 82.57$ & $266.66 \pm 140.12$ & 0.016 \\
\hline TP & $575.54 \pm 529.71$ & $\begin{array}{c}1019.29 \pm 703.2 \\
5\end{array}$ & 0.018 \\
\hline \multicolumn{4}{|c|}{ Table 2: Comparison of Geometrical Parameters of the } \\
PCOS and Control Group \\
\hline
\end{tabular}

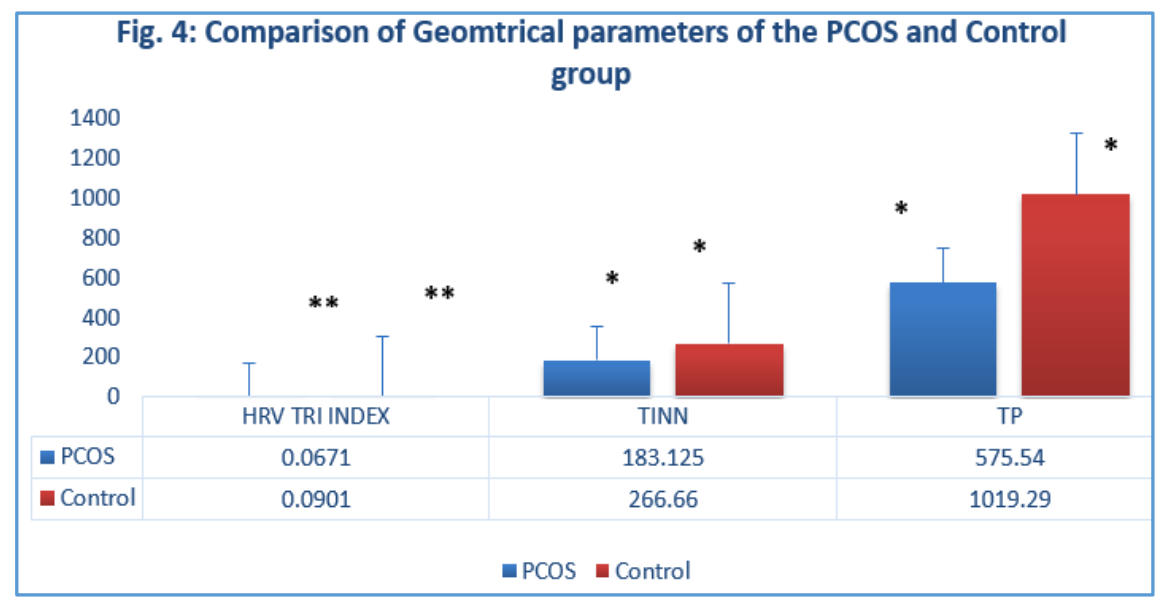

Fig. 4: HRV TRI INDEX-Density Distribution, TINN- Triangular Interpolation of NN Interpolation of NN Interval Histogram, TP- Total Power. **- Moderately Significant. *- Significant

\begin{tabular}{|c|c|c|c|c|c|c|c|c|c|}
\hline Parameters & & SD1 & SD2 & SD1/SD2 & S & TRI Index & TINN & TP & BMI \\
\hline RPP & r value & 0.640 & -.496 & -.505 & -.523 & -.628 & -.407 & -.446 & 0.114 \\
\hline & p value & 0.000 & 0.000 & 0.000 & 0.000 & 0.000 & 0.004 & 0.001 & 0.442 \\
\hline BMI & r value & -.160 & -.176 & -.098 & -.162 & -.288 & -.124 & -.193 & \\
\hline & p value & 0.277 & 0.232 & 0.506 & 0.271 & 0.047 & 0.400 & 0.189 & \\
\hline
\end{tabular}

\begin{tabular}{|c|c|c|c|c|}
\hline \multirow{2}{*}{ Parameters } & & TINN & HRV TRI Index & TP \\
\hline \multirow{2}{*}{$\mathrm{S}$} & r value & 0.813 & 0.814 & .895 \\
\cline { 2 - 5 } & p value & 0.000 & 0.000 & 0.000 \\
\hline \multirow{2}{*}{ TP } & r value & 0.681 & 0.820 & \\
\cline { 2 - 5 } & p value & 0.000 & 0.000 & \\
\hline \multicolumn{2}{|c|}{ Table 4: Correlation Analysis between HRV Parameters } \\
\hline
\end{tabular}

\begin{tabular}{|c|c|c|c|c|c|c|c|c|}
\hline Parameters & & SD1 & SD2 & SD1/SD2 & S & TRI Index & TINN & TP \\
\hline RPP & Standardised beta & -1.792 & -1.969 & -.058 & 2.019 & -.922 & 1.617 & 1.133 \\
\hline & p value & 0.81 & 0.056 & 0.954 & 0.050 & 0.362 & 0.114 & 0.264 \\
\hline BMI & Standardised beta & 0.973 & -.189 & -.851 & -.052 & -1.753 & -.715 & -.256 \\
\hline & p value & 0.337 & 0.851 & 0.400 & 0.959 & 0.087 & 0.479 & 0.799 \\
\hline
\end{tabular}




\section{DISCUSSION}

PCOS is a disorder of present day which requires keen observation and treatment as it is with chronic anovulation, hyperandrogenism, hirsutism, obesity, sub-fertility and insulin resistance. They have high incidence of hypertension.(28) and CVD.(29) Studies show that among the PCOS, $50 \%$ of women have insulin resistance and no more than $40 \%$ are obese. ${ }^{(30)}$

Obesity is a condition characterised by hyperinsulinaemia and insulin resistance.(31) and is associated with increased incidence of HT.(32) and enhanced risk for cardiovascular morbidity.(33) Obesity has long been known to cause alterations in autonomic functions in the form of increased adrenergic and decreased vagal modulation.(14)

Therefore, we selected our study group population without obesity. They were of lean and ideal BMI and weight category and they were young. Since we excluded the people with obesity, DM and hypertension, influence of these pathology on cardiac autonomic modulation is prevented.

On looking into the cardiovascular parameters though their systolic and diastolic resting blood pressure was normal, RPP was significantly higher among the PCOS than the controls. (Table 1; Fig. 2) RPP denotes increased oxygen demand and increased myocardial load.(24) Therefore, PCOS patients are under increased stress on the myocardium. Also basal heart rate was significantly higher among the PCOS than the control.

HRV is a powerful tool to 'evaluate cardiac autonomic activity. Diminished HRV is associated with increased sympathetic and decreased vagal modulation and these autonomic changes have been reported to be associated with an increase in the malignant ventricular arrhythmias.(4) We looked into 3 categories of HRV parameters to analyse in this study. They are the Geometric methods (RR INDEX, TINN), the non-linear Poincare Plot measures (SD1, SD2, SD1/SD2 and S) and in spectral analysis (TP). The time domain measures and spectral analysis were dealt in detail in our previous study.(17)

In frequency domain analysis, TP which is an index of overall HRV was significantly reduced in the cases (Table 2; Fig. 4). Decreased HRV depicts decreased cardiovagal modulation, which is a potential CV risk.(34) TP showed a significant negative association with RPP and not associated with BMI. The geometric parameters HRV TRI INDEX and TINN, which express overall HRV measured over 24 hours and are more influenced by lower than higher frequency of HRV spectrum.(16) were significantly reduced (Table 2; Fig. 4), favouring sympathovagal imbalance. Both significantly correlated with RPP, but not with BMI (Table 3).

The Poincare parameters SD1, SD2, SD1/SD2 was not significantly altered. (Table 1 ; Fig. 2) It could be said that short- and long-term variability was not observed, but Area $S$ which is the total area of the ellipse denoting total variability. ${ }^{(27)}$ was reduced significantly in PCOS than controls. (Table 1; Fig. 3) Negative correlation of RPP non-linear measures, SD1, SD2, SD1/SD2 and S depicts that attenuated total variability could increase the stress on myocardial performance (Table 3).

TP and S which represents total variability has positive correlation with reduced geometric measures as well as TP and S correlate positively. This shows they are interchangeable. The regression analysis did not lay forward the independent associations of these variables (Table 4).
This study indicates the total variability is decreased even in lean and ideal weight PCOS patients supporting our previous study, which analysed time and frequency domain measures alone and found altered HRV parameters.(17) This shows altered cardiac autonomic activity even in lean and ideal weight PCOS, which is a risk factor for cardiovascular disease.

\section{LIMITATIONS OF THE STUDY}

One of the main limitations is the small sample size of study groups. Second is that we were not able to do a direct comparative analysis between obese and lean PCOS and the last is that lean and ideal weight PCOS were categorized based on BMI and not on absence of visceral obesity (According to waist - hip ratio).

\section{ACKNOWLEDGEMENT}

We are thankful to Dr. R. Nagashree, Professor and Head, Department of Physiology, Dr. Alice Kuruvilla , Department of Pharmacology, Dr. K. Bhuvaneswari, Department of Pharmacology, Dr. Saira Banu, Department of Community Medicine, Dr. Anand and Dr. Usha Anand, department of Biochemistry and Mr.Mohammed Ansari for their valuable guidance and support.

\section{CONCLUSION}

The result of the present study shows that cardiac autonomic activity can be affected in PCOS, overall decrease in geometric and total variability of HRV. Therefore, all PCOS irrespective of obesity and age should be screened for cardiac dysfunction and routine investigations should include ECG with HRV.

\section{REFERENCES}

1. Zargar AH, Gupta VK, Wani AI, et al. Prevalence of ultrasonography proven polycystic ovaries in North Indian women with type 2 diabetes mellitus. Reproductive Biology and Endocrinology 2005;3:35.

2. Ovalle F, Ricardo A. Insulin resistance, polycystic ovary syndrome and type II diabetes mellitus. Fertil Steril 2002;77(6):1095-105.

3. Wild S, Pierpoint T, McKeigue P, et al. Cardiovascular disease in women with polycystic ovarian syndrome at a long term follow up: a retrospective cohort study. Clin Endocrinol (Oxf) 2000;52(5):595-600.

4. Dahlgren E, Janson PO, Johansson S, et al. Polycystic ovary syndrome and risk for myocardial infarction: evaluated from a risk factor model based on a prospective population study of women. Acta Obstet Gynaecol Scand 1992;71(8):599-604.

5. Carmine E, Lobo RA. Polycystic ovary syndrome (PCOS): arguably the most common endocrinopathy is associated with significant morbidity in women. The Journal of clinical Endocrinology and Metabolism 1999;84(6):1897-9.

6. Prelevic GM, Beijic T, Balint-Peric L, et al. Clinical endocrinology. Cardiac flow velocity in women with polycystic ovary syndrome. Clin Endocrinol 1995;43:67781.

7. Yarali H, Yildirir A, Aybar F, et al. Diastolic dysfunction and increased serum homocysteine concentrations may contribute to increased cardiovascular risk in patients with polycystic ovary syndrome. Fertil Steril 2001;76(3):511-6. 
8. Kelly CJ, Speirs A, Gould GW, et al. Altered vascular function in young women with polycystic ovary syndrome. J Clin Endocrinol Metab 2002;87(2):742-6.

9. Paradisi G, Steinberg HO, Hempfling A, et al. Polycystic ovary syndrome is associated with endothelial dysfunction. Circulation 2001;103:1410-5.

10. Kelly CC, Lyall H, Petrie JR, et al. Low grade chronic inflammation in women with polycystic ovary syndrome. J Clin Endocrinol Metab 2001;86(6):2453-5.

11. Loverro G, Lorusso F, Mei L, et al. The plasma homocysteine levels are increased in polycystic ovary syndrome. Gynaecol Obstet Invest 2002;53(3):157-62.

12. Yildiz BO, Haznedaroglu IC, Kirazli S, et al. Global fibrinolytic capacity is decreased in polycystic ovary syndrome, suggesting a prothrombic state. J Clin Endocrinol Metab 2002;87(8):3871-5.

13. Kelly CJ, Lyall H, Petrie JR, et al. A specific elevation in tissue plasminogen activator antigen in women with polycystic ovary syndrome. J Clin Endocrinol Metab 2002;87(7):3287-90.

14. Seals DR, Bell C. Chronic sympathetic activation: consequence and cause of age-associated obesity? Diabetes 2004;53(2):276-84.

15. Balamurugan $M$, Balamurugan, Ramanathan G. Metabolic syndrome in lean Indian women with polycystic ovary syndrome. International Journal Of Medical Science and Research 2009;2(3):37-46.

16. Malik M. Heart rate variability. Standards of measurement, physiological interpretation, and clinical use. Task force of the European Society of Cardiology the North American Society of Pacing Electrophysiology. American Heart Association, Circulation 1996;93:1043-65.

17. Balamurugan M, Balamurugan M, Ramanathan G. Heart rate variability and lipid profile in non-obese young Indian women with polycystic ovary syndrome. Journal of Evolution of Medical and Dental Sciences 2015;4(24):4092-109.

18. Voss A, Kurths J, Kleiner HJ, et al. Improved analysis of heart rate variability by methods of nonlinear dynamics. J Electrocardiol 1995;28 Suppl:81-8.

19. Piskorski J, Guzik P. Geometry of the poincaré plot of RR intervals and its asymmetry in healthy adults. Physiol Meas 2007;28(3):287-300.

20. The Rotterdam ESHRE/ASRM-sponsored PCOS consensus workshop group. Revised 2003 consensus on diagnostic criteria and long term health risks related to polycystic ovary syndrome. Fertility and sterility 2004;81(1):19-25.
21. Ota T, Takamura T, Hirai N, et al. BMI classification physical status: preobesity in world health organization classification involves the metabolic syndrome in Japanese. Diabetes Care 2002;25(7):1252-3.

22. World Health Organization. Obesity-preventing and managing the global epidemic: report of a WHO consultation on obesity. Geneva: World Health Organization, 1998.

23. Lean ME, Han TS, Morrison CE. Waist circumference as a measure for indicating need for weight management. BMJ 1995;311(6998):158-61.

24. White WB. Heart rate and the rate-pressure product as determinants of cardiovascular risk in patients with hypertension. Am J Hypertens 1999;12(2 Pt 2):50S-5.

25. Stein PK, Reddy A. Non-linear heart rate variability and risk stratification in cardiovascular disease. Indian Pacing Electrophysiol J 2005;5(3):210-20.

26. Brennan M, Palaniswami M, Kamen P. Do existing measures of poincarè plot geometry reflect nonlinear features of heart rate variability? IEEE Trans Biomed Eng 2001;48(11):1342-7.

27. Guzik P, Bychowiec B, Piskorski J, et al. Heart rate variability by poincaré plot and spectral analysis in young healthy subjects and patients with type 1 diabetes. Folia Cardiol 2005;12 Suppl D:64-7.

28. Dahlgren E, Janson PO, Johansson S, et al. Haemostatic and metabolic variables in women with polycystic ovary syndrome. Fertil Steril 1994;61(3):455-60.

29. Holmang A, Svedberg J, Jennische E, et al. Effects of testosterone on muscle insulin sensitivity and morphology in female rats. Am J Physiol Endocrinol Metab 1990;259(4 Pt 1):E555-60.

30. Hemmings R, Farookhi R, Brawer JR. Pituitary and ovarian responses to LHRH in a rat with polycystic ovaries. Biol Reprod 1983;29(1):239-48.

31. Ferranni E, Natali $A$, Bell $P$, et al. Insulin resistance and hypertension in obesity. European group for the study of insulin resistance. J Clin Invest 1997;100(5):1166-73.

32. Ward KD, Sparrow D, Landsberg L, et al. Influence of insulin, sympathetic nervous system activity and obesity on blood pressure: the normative aging study. J Hypertens 1996;14(3):301-8.

33. Calle EE, Thun MJ, Petrelli JM, et al. Body mass index and mortality in a prospective cohort of U.S adults. N Engl J med 1999;341(15):1097-105.

34. Thayer JF, Yamamoto SS, Brosschot JF. The relationship of autonomic imbalance, heart rate variability and cardiovascular disease risk factors. Int $\mathrm{J}$ Cardiol 2010;141(2):122-31. 